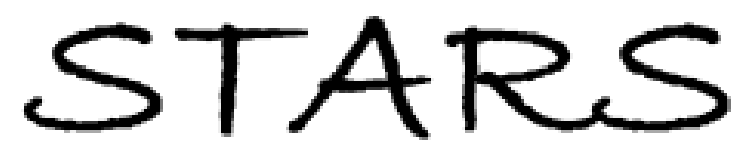

University of Central Florida

STARS

$1-1-2013$

\title{
Optical rotatory power of polymer-stabilized blue phase liquid crystals
}

\author{
Yifan Liu \\ University of Central Florida \\ Yi-fen Lan \\ University of Central Florida \\ Hongxia Zhang \\ University of Central Florida \\ Ruidong Zhu \\ University of Central Florida \\ Daming $\mathrm{Xu}$ \\ University of Central Florida
}

Find similar works at: https://stars.library.ucf.edu/facultybib2010

University of Central Florida Libraries http://library.ucf.edu

See next page for additional authors

This Article is brought to you for free and open access by the Faculty Bibliography at STARS. It has been accepted for inclusion in Faculty Bibliography 2010 s by an authorized administrator of STARS. For more information, please contactSTARS@ucf.edu.

\section{Recommended Citation}

Liu, Yifan; Lan, Yi-fen; Zhang, Hongxia; Zhu, Ruidong; Xu, Daming; Tsai, Cheng-Yeh; Lu, Jen-Kuei; Sugiura, Norio; Lin, Yu-Chieh; and Wu, Shin-Tson, "Optical rotatory power of polymer-stabilized blue phase liquid crystals" (2013). Faculty Bibliography 2010s. 4326.

https://stars.library.ucf.edu/facultybib2010/4326

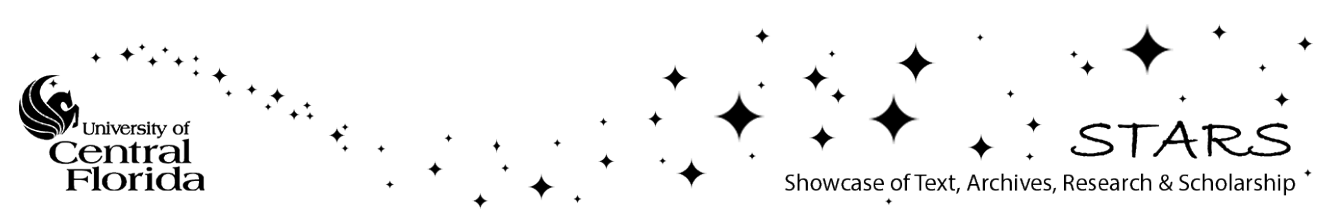




\section{Authors}

Yifan Liu, Yi-fen Lan, Hongxia Zhang, Ruidong Zhu, Daming Xu, Cheng-Yeh Tsai, Jen-Kuei Lu, Norio Sugiura, Yu-Chieh Lin, and Shin-Tson Wu 


\section{Optical rotatory power of polymer-stabilized blue phase liquid crystals}

Cite as: Appl. Phys. Lett. 102, 131102 (2013); https://doi.org/10.1063/1.4799511

Submitted: 23 January 2013 . Accepted: 20 March 2013 . Published Online: 01 April 2013

Yifan Liu, Yi-fen Lan, Hongxia Zhang, Ruidong Zhu, Daming Xu, Cheng-Yeh Tsai, Jen-Kuei Lu, Norio Sugiura, Yu-Chieh Lin, and Shin-Tson Wu
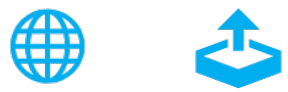

\section{ARTICLES YOU MAY BE INTERESTED IN}

A large Kerr constant polymer-stabilized blue phase liquid crystal

Applied Physics Letters 98, 081109 (2011); https://doi.org/10.1063/1.3559614

Extended Kerr effect of polymer-stabilized blue-phase liquid crystals

Applied Physics Letters 96, 071105 (2010); https://doi.org/10.1063/1.3318288

Low voltage blue-phase liquid crystal displays

Applied Physics Letters 95, 231101 (2009); https://doi.org/10.1063/1.3271771

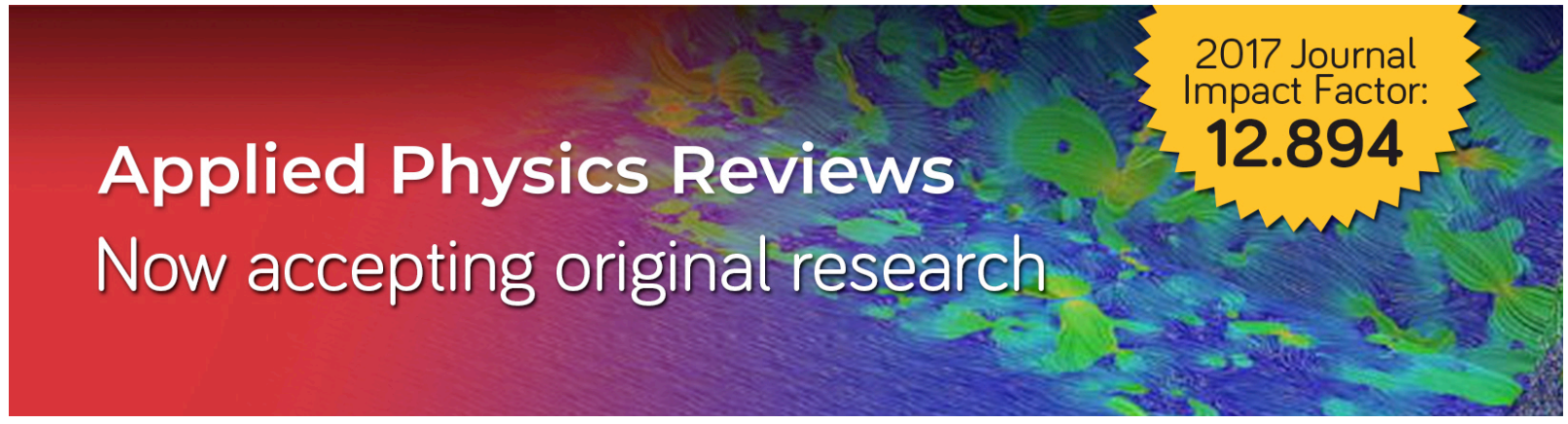




\title{
Optical rotatory power of polymer-stabilized blue phase liquid crystals
}

\author{
Yifan Liu, ${ }^{1}$ Yi-fen Lan, ${ }^{1,2}$ Hongxia Zhang, ${ }^{1,3}$ Ruidong Zhu, ${ }^{1}$ Daming Xu, ${ }^{1}$ Cheng-Yeh Tsai, ${ }^{2}$ \\ Jen-Kuei Lu, ${ }^{2}$ Norio Sugiura, ${ }^{2}$ Yu-Chieh Lin, ${ }^{2}$ and Shin-Tson Wu ${ }^{1, a)}$ \\ ${ }^{1}$ College of Optics and Photonics, University of Central Florida, Orlando, Florida 32816, USA \\ ${ }^{2}$ AU Optronics Corp., Hsinchu Science Park, Hsinchu 300, Taiwan \\ ${ }^{3}$ Tianjin University, 92 Weijin Road, Nankai District, Tianjin 300072, China
}

(Received 23 January 2013; accepted 20 March 2013; published online 1 April 2013)

\begin{abstract}
Macroscopically, a polymer-stabilized blue phase liquid crystal (BPLC) is assumed to be an optically isotropic medium. Our experiment challenges this assumption. Our results indicate that the optical rotatory power (ORP) of some nano-scale double-twist cylinders in a BPLC composite causes the polarization axis of the transmitted light to rotate a small angle, which in turn leaks through the crossed polarizers. Rotating the analyzer in azimuthal direction to correct this ORP can greatly improve the contrast ratio. A modified De Vries equation based on a thin twisted-nematic layer is proposed to explain the observed phenomena. (C) 2013 American Institute of Physics. [http://dx.doi.org/10.1063/1.4799511]
\end{abstract}

A polymer-stabilized blue phase liquid crystal (PSBPLC) is a self-assembly nanostructure electro-optic medium. ${ }^{1-7}$ The self-assembly process forms three dimensional lattices without the need of surface alignment agent, and the voltage-off state is optically isotropic. The short coherence length leads to submillisecond response time. ${ }^{6,7}$ Thus, BPLC holds potential for high speed display and photonic devices. However, some critical issues, such as relatively high operation voltage, noticeable hysteresis, and limited contrast ratio (CR), remain to be overcome. Several promising approaches for reducing operation voltage $e^{8-10}$ and suppressing hysteresis ${ }^{11,12}$ have been demonstrated, but the problem of CR remains unexplored. Most nematic liquid crystal display (LCD) devices can reach CR > 5000:1, but BPLCs can barely reach $\sim 1000: 1{ }^{5,13}$ The low CR of a PS-BPLC mainly originates from the dark state light leakage. There is an urgent need to understand the light leakage mechanism and then improve the contrast ratio.

Macroscopically, a PS-BPLC is commonly treated as an optically isotropic medium. ${ }^{1,4}$ Based on this assumption, an excellent dark state (or very high CR) should be obtained when a BPLC cell is sandwiched between two crossed polarizers. But if this is true, then where does the light leakage come from? Some factors affecting the dark state light leakage have been investigated, such as Bragg reflection ${ }^{13}$ and light refraction on the edge of ITO (indium tin oxide) electrodes. ${ }^{14}$ Undoubtedly, these are important factors, but still another fundamental mechanism should be taken into consideration, which is optical rotatory power (ORP). ${ }^{15-18}$ According to Meiboom's model, ${ }^{19}$ BPLC is a three-dimensional structure consisting of double-twist cylinders whose diameter is around $100 \mathrm{~nm}$. Inside each cylinder, LC molecules form doubletwist structure. When the incident linearly polarized light traverses these cylinders, a small ORP could be accumulated depending on the cylinder's orientation. As a result, the polarization state of the outgoing light could be rotated by a small angle, which in turn leaks through the crossed polarizer and degrades the CR significantly.

${ }^{a)}$ Electronic mail: swu@ucf.edu
In this Letter, we characterize the ORP of PS-BPLC samples with different Bragg reflection wavelengths $\left(\lambda_{\mathrm{B}}\right)$. Some double-twist cylinder orientations within a BPLC composite indeed cause the polarization axis of the transmitted beam to rotate and leak through the crossed polarizers. Rotating the analyzer in azimuthal direction to correct the ORP boosts the white light $\mathrm{CR}$ of the device by $3-5 \times$. A modified De Vries equation based on a thin twist-nematic (TN) layer ${ }^{20}$ is proposed to explain this phenomenon.

Our BPLC precursors consist of four ingredients: (1) nematic LC host (HTG135200-100), (2) right-handed chiral dopant (R5011); both are from HCCH China; (3) di-functional reactive monomer RM257 (Merck), and (4) mono-functional reactive monomer C12A (Sigma Aldrich). Detailed preparation process has been reported in Ref. 21. To obtain different Bragg reflection wavelengths, we varied the weight ratios of LC host and chiral dopant while fixing the monomer weight ratios at 6 wt. \% RM257 and 4 wt. \% C12A. The precursors were cured in the BP-I phase with a UV light $(\lambda \sim 365 \mathrm{~nm}$ and intensity $\sim 8 \mathrm{~mW} / \mathrm{cm}^{2}$ ) for $10 \mathrm{~min}$.

The experimental setup for characterizing the optical rotary power of a BPLC cell is described as follows: Three laser wavelengths were used for measurement: $R=633 \mathrm{~nm}$, $\mathrm{G}=514 \mathrm{~nm}$, and $\mathrm{B}=457 \mathrm{~nm}$. The transmission axis of the front linear polarizer was fixed, while the analyzer was crossed to the polarizer initially. The BPLC sample was sandwiched between the polarizers, and the light leakage measured. Without a sample, the extinction ratio of the crossed polarizers exceeds $10^{5}: 1$.

We first investigated the BPLC cell with in-plane switching (IPS) electrodes. The electrode width/gap was $8 \mu \mathrm{m} / 12$ $\mu \mathrm{m}$, and cell gap $d$ was $7.4 \mu \mathrm{m}$. The upper half of Table I lists the measured transmittance $\left(\mathrm{T}_{\perp}\right)$ of this sample at the specified red-green-blue (RGB) wavelengths, when the analyzer was crossed. Surprisingly, $T_{\perp}$ is still quite noticeable. But if we rotate the analyzer in azimuthal direction by a small angle $\alpha$, a much smaller $T_{\perp}^{\prime}$ can be achieved for each wavelength, implying the outgoing light still keeps a fairly good linear polarization, but its polarization axis is rotated by $\alpha$. 
TABLE I. Polarization rotation effect: Comparison of IPS and VFS samples. $\mathrm{T}_{\perp}$ : Transmittance with analyzer crossed. $T_{\perp}^{\prime}$ : Transmittance with analyzer rotated by an angle $\alpha$. Here, the positive $\alpha$ results from the employed righthanded chiral dopant.

\begin{tabular}{lcccc}
\hline \hline$\lambda(\mathrm{nm})$ & $\mathrm{T}_{\perp}(\%)$ & $\alpha(\mathrm{deg})$ & $\alpha / \mathrm{d}(\mathrm{deg} / \mu \mathrm{m})$ & $T_{\perp}^{\prime}(\%)$ \\
\hline \multicolumn{5}{c}{ IPS cell, $\mathrm{d}=7.4 \mu \mathrm{m}$} \\
633 & 0.02 & 0.5 & 0.07 & 0.004 \\
514 & 0.10 & 2.0 & 0.27 & 0.020 \\
457 & 0.60 & 4.0 & 0.54 & 0.090 \\
& 0.02 & VFS cell, d = 10 $\mu \mathrm{m}$ & \\
633 & 0.20 & 0.5 & 0.05 & 0.004 \\
514 & 1.0 & 2.5 & 0.25 & 0.020 \\
457 & 5.5 & 0.55 & 0.080 \\
\hline \hline
\end{tabular}

This polarization rotation could originate from both BPLC material and light refraction at the edges of electrode; the latter has been reported in Ref. 14. To rule out the refraction effect and focus on the BPLC material property, we use the vertical-field switching (VFS) cell, which was made of two planar ITO glass substrates without polyimide layer. ${ }^{22,23}$ The cell gap was $d=10 \mu \mathrm{m}$. Similar to an IPS cell, we measure the light leakage of VFS sample. The results are included in the lower half of Table I. We find that crossed analyzer does not lead to the darkest state for either IPS or VFS cell. Rotating a small angle $\alpha$ can reduce the light leakage by $5-10 \times$, depending on the wavelength. The VFS and IPS cells have similar $\alpha / d$ and $T_{\perp}^{\prime}$ values, indicating that these two cells have a similar optical rotatory property, resulting from the BPLC material property, not from the refraction on ITO electrode edge.

To focus on the material property, in the following experiments, we use VFS cells for all the measurements. Considering that outgoing light still keeps a good linear polarization, the polarization rotation angle caused by BPLC is the same as the analyzer rotation angle $(\alpha)$ from crossed position. ORP, $\varphi \equiv \alpha / d$, is defined as the polarization rotation angle divided by the cell gap. Unless otherwise mentioned, all measurements were conducted at room temperature $\left(20{ }^{\circ} \mathrm{C}\right)$.

In experiment, we first investigate how the temperature affects ORP. We prepared a BPLC sample, designated as HTG-23, using the above mentioned recipes. Its Bragg reflection wavelength is $\lambda_{\mathrm{B}} \sim 410 \mathrm{~nm}$ and clearing point after $\mathrm{UV}$ curing is $78{ }^{\circ} \mathrm{C}$. We measured its ORP at $\lambda=514 \mathrm{~nm}$ and $457 \mathrm{~nm}$ between $30{ }^{\circ} \mathrm{C}$ and $90{ }^{\circ} \mathrm{C}$, and results are plotted in Figs. 1(a) and 1(b), respectively. In Fig. 1, circles represent the measured ORP values. The BPLC sample was placed on a rotary mount whose scale is $2^{\circ}$ per division, so the precision of our data is $\pm 0.5^{\circ}$ in azimuthal angle. Therefore, the data shown in Fig. 1 appear stepwise. A positive ORP implies that polarization rotation is in the right-hand direction.

To understand the temperature dependent ORP data in Fig. 1, we measured the temperature dependent induced birefringence of the PS-BPLC, which in principle can be estimated from ${ }^{24}$

$$
\Delta n_{s}=\Delta n_{0} C\left(1-T / T_{c}^{\prime}\right)^{\beta}
$$

where $\Delta n_{s}$ is the saturated birefringence of Kerr-effectinduced isotropic-to-anisotropic transition in the BPLC, ${ }^{25} \Delta n_{0}$
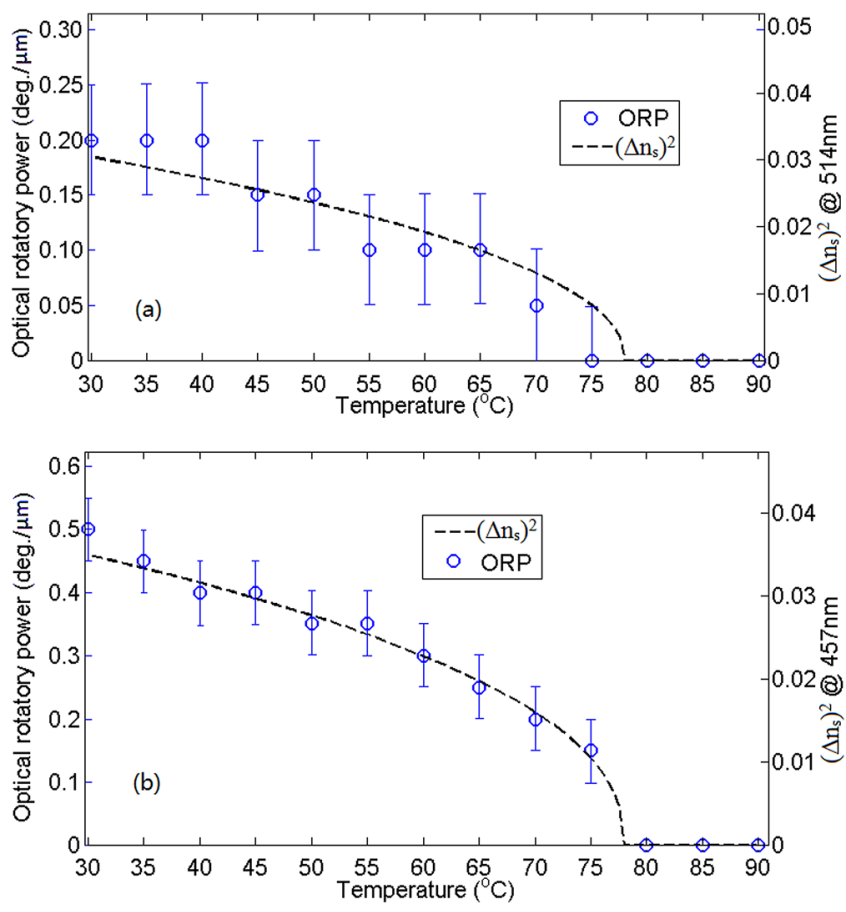

FIG. 1. Temperature dependent ORP (left) and $\left(\Delta \mathrm{n}_{\mathrm{s}}\right)^{2}$ (right) of HTG-23.

is the extrapolated birefringence of the $\mathrm{LC}$ host at $\mathrm{T}=0, \mathrm{C}$ is the $\mathrm{LC}$ host concentration (wt. \%) in the composite, $\beta$ is a material parameter, and $T_{c}^{\prime}$ is the clearing point of BPLC after UV curing. Using the measured data (not shown here), we obtained $\beta=0.227$, and $\Delta n_{0}=0.296,0.321$, and 0.347 for $\lambda=633 \mathrm{~nm}, 514 \mathrm{~nm}$, and $457 \mathrm{~nm}$, respectively. Dashed lines in Fig. 1 represent the temperature dependent $\left(\Delta n_{\mathrm{s}}\right)^{2}$. Semiempirically, we found that ORP correlates with $\left(\Delta \mathrm{n}_{\mathrm{s}}\right)^{2}$ reasonably well

$$
\varphi \propto\left(\Delta n_{s}\right)^{2}
$$

In total, we prepared 15 PS-BPLC samples with different $\lambda_{B}$ 's, and measured their ORP at the specified RGB laser wavelengths. Results are plotted in Fig. 2. In Fig. 2, red open circles, green solid circles, and blue triangles represent the measured ORP of BPLC samples at $\lambda=633 \mathrm{~nm}, 514 \mathrm{~nm}$, and $457 \mathrm{~nm}$, respectively, while red solid line, green dashed lines, and blue dotted lines are the corresponding fitting curves. The employed fitting equation will be discussed later. From Fig. 2, when $\lambda_{B}$ increases towards the incident light wavelength $\lambda$, ORP increases rapidly. However, when $\lambda_{B}$ gets closer to or longer than $\lambda$, ORP becomes difficult to measure because scattering dominates the transmitted light, and it is difficult to determine $\varphi$ accurately.

The fast rising of ORP with $\lambda_{B}$ can also be found in cholesteric liquid crystal as described by the De Vries equation. $^{26}$ De Vries equation has a term $\left(\lambda^{2} / \lambda_{B}^{2}-1\right)$ in the denominator, in which $\lambda_{B}$ represents the Bragg reflection wavelength of a cholesteric LC. Therefore, it is reasonable to assume the equation of ORP in BPLC also has the same term in the denominator.

Finally, based on the above experimental results, we select the following equation to fit the ORP of our BPLC samples 


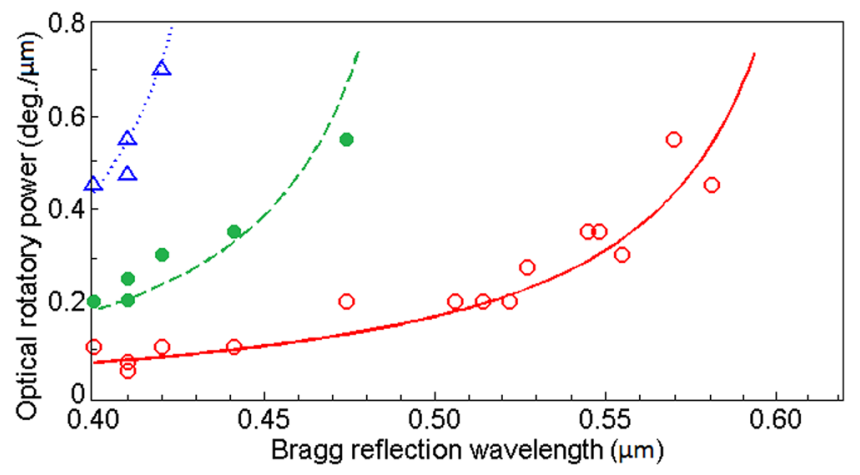

FIG. 2. Bragg reflection wavelength vs. optical rotatory power: red open circles are the measured ORP at $\lambda=633 \mathrm{~nm}$, green filled circles at $\lambda=514 \mathrm{~nm}$, and blue triangles at $\lambda=457 \mathrm{~nm}$. Lines are fitting curves using Eq. (3) with only one parameter $\varphi_{\mathrm{o}}=3.12 \mathrm{deg} / \mu \mathrm{m}$.

$$
\varphi=\varphi_{o} \frac{\left(\Delta n_{s}\right)^{2}}{\lambda^{2} / \lambda_{B}^{2}-1} .
$$

In Eq. (3), $\varphi_{0}$ is a fitting parameter, $\Delta n_{s}$ is the saturated birefringence of BPLC, which is a function of wavelength and temperature, and $\lambda_{B}$ is the Bragg reflection wavelength. We use Eq. (3) to fit the measured ORP data, as Fig. 2 shows. With only one fitting parameter $\left(\varphi_{0}=3.12 \mathrm{deg} / \mu \mathrm{m}\right)$, good agreement between experiment and Eq. (3) is obtained.

Figure 2 shows that the ORP of BPLC samples is in the $0.1^{\circ} / \mu \mathrm{m}$ to $0.7^{\circ} / \mu \mathrm{m}$ range. Since a typical BPLC cell gap is $5-10 \mu \mathrm{m}$, the analyzer could deviate by $0.5^{\circ}$ to $7^{\circ}$ from the perfectly crossed position. To see how this small deviation angle affects the device contrast ratio, we tested the BPLC samples HTG-23 again using the same experimental setup: First, we set the analyzer crossed to the polarizer. Then, we rotated the analyzer in azimuthal direction to minimize the light leakage for each wavelength. Result are shown in Fig. 3.

In Fig. 3, the horizontal axis shows the analyzer rotation angle $\left(0^{\circ}\right.$ means the analyzer is crossed) and vertical axis represents the light leakage percentage (in logarithmic scale). Red open circles, green filled circles, and blue triangles are light leakage at $\lambda=633 \mathrm{~nm}, 514 \mathrm{~nm}$, and $457 \mathrm{~nm}$,

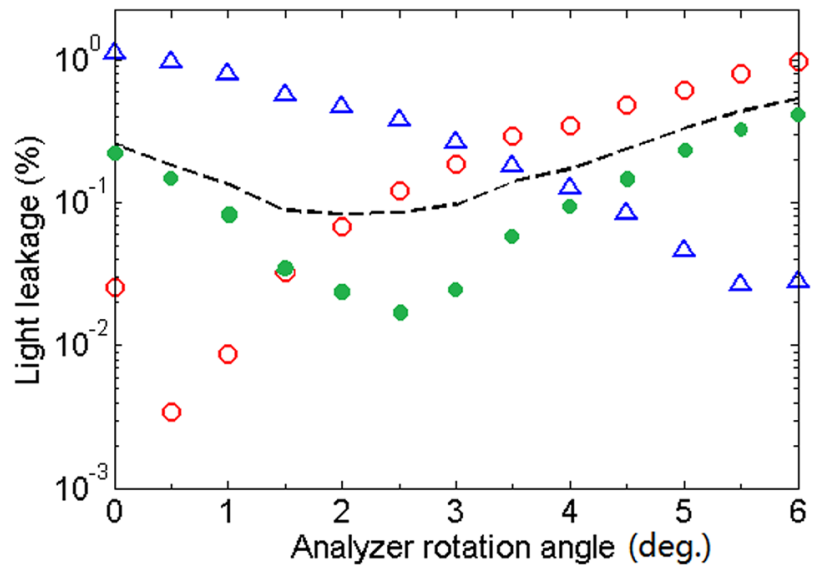

FIG. 3. Light leakage vs. analyzer rotation angle. Here, $0^{\circ}$ means the analyzer is crossed with polarizer, and positive rotation angle represents rotation in right-hand direction. Red open circles are the measured light leakage at $\lambda=633 \mathrm{~nm}$, green filled circles at $\lambda=514 \mathrm{~nm}$, and blue triangles at $\lambda=457 \mathrm{~nm}$. Dashed lines are the overall leakage of white light, including $30 \%$ red, $60 \%$ green, and $10 \%$ blue lights. respectively. From Fig. 3, the dark state light leakage can be reduced by $\sim 10 \times$ for a given wavelength by adjusting the analyzer's angle. However, each wavelength has its own optimal analyzer angle. For LCDs, a white backlight is usually comprised of $60 \%$ green, $30 \%$ red, and $10 \%$ blue spectral contents. We take these ratios into account and calculate the overall white light leakage as the black dashed lines shown in Fig. 3. When the analyzer is crossed, white light leakage is $\sim 0.25 \%$, corresponding to $\mathrm{CR} \sim 320: 1$, assuming the bright state transmittance is $80 \%$ for a typical IPS cell. If we rotate the analyzer by $\sim 2^{\circ}$, the white light leakage drops to $\sim 0.08 \%$, and the CR is boosted to $1000: 1$. Moreover, based on Eq. (3), if we shift $\lambda_{B}$ from $410 \mathrm{~nm}$ to $350 \mathrm{~nm}$ (this is a common practice for a clear BPLC), the overall leakage would decrease noticeably, and the white light contrast ratio would exceed 3000:1.

Next, we try to explain the observed optical rotatory power by a thin TN model. It is known that in BP-I phase, the LC molecules are arranged in double-twist cylinders, as sketched in Fig. 4, and these cylinders are stacked to form body-centered cubic (BCC) structure. Let us assume the incident light is in the positive $z$ direction in Fig. 4, the optical activity of each cylinder is illustrated too. Due to the random alignment of BCC domains, the incident light could propagate along any direction into the cylinders. But for the simplicity of discussion, we focus on the basic configurations, when the incident wave vector is either parallel or perpendicular to the cylinder's axis. On the left side of Fig. 4, the incident light propagates along the cylinder's axis. Because the LC molecules in the cylinder are aligned equally in all directions, the cylinder appears optically isotropic, which does not affect the polarization state of the incident light. However, for the other two cylinders in Fig. 4, the light propagation directions are perpendicular to their axis. Therefore, as the light traverses the cylinder, it will interact with the $90^{\circ}$ twisted LC directors. This twisted alignment affects the outgoing polarization of the light. Generally speaking, the outgoing light will be elliptically polarized, with the long axis of this ellipse rotated by a certain angle $\theta$ away from incident polarization direction, as shown in Fig. 4. This phenomenon is similar to the case when the light passing through a very thin TN cell. Thus, we simulate the optical activity of a TN cell by Jones matrix and see if we can find analogy between TN and BPLC.
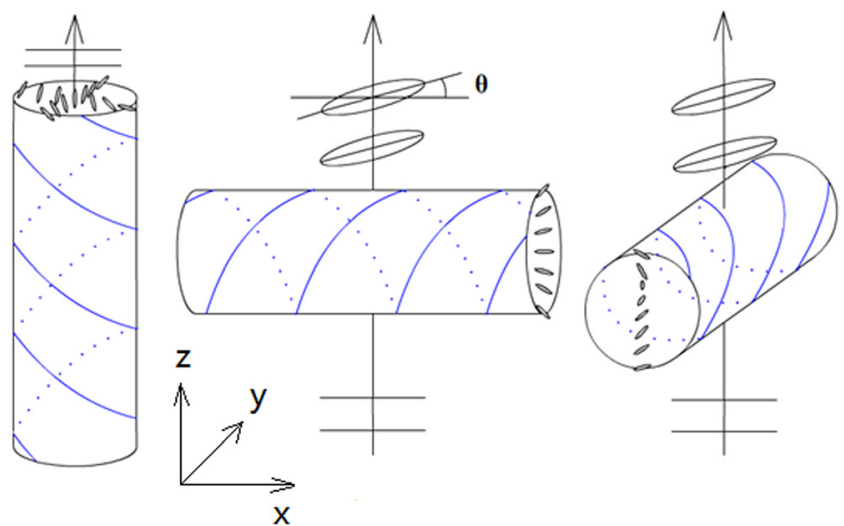

FIG. 4. LC director configuration in BP-I phase: Double-twist cylinders. 


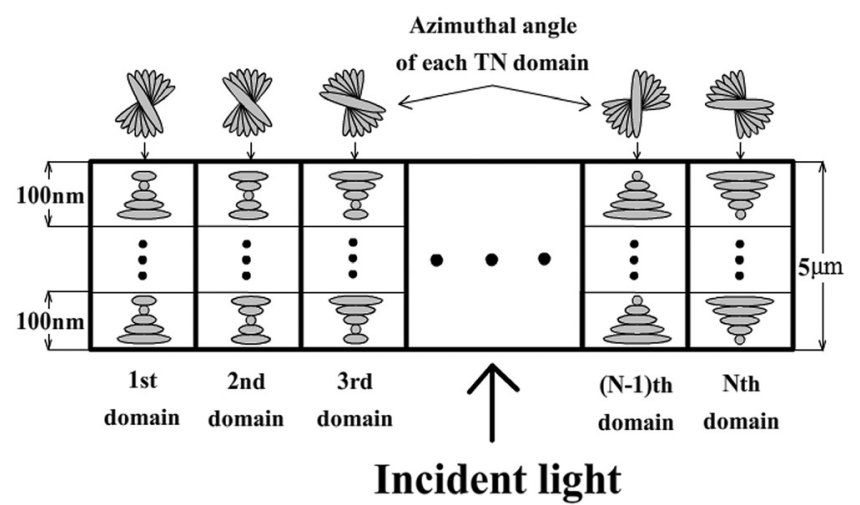

FIG. 5. The multi-domain multi-layer TN cell structure used in the simulation. Upper part: top view, and lower part: cross-section view.

Considering that in a VFS cell, there are multiple microdomains of BPLC, aligning in different directions. Besides, the BCC structure of BPLC has multiple layers of doubletwist cylinders stacked inside each domain. Therefore, to imitate the optical activity of BPLC precisely, we use a multi-domain, multi-layer TN LC cell model, as shown in Fig. 5. We divide a 5- $\mu$ m-thick LC cell into $N(N>100)$ domains. Each aligned in a random azimuthal angle, and divided into multiple layers. Each layer is a 100-nm-thick TN cell with $90^{\circ}$ twist angle in right hand direction. The LC birefringence is 0.2 , and the wavelength is $550 \mathrm{~nm}$.

We simulate the polarization of the light passing through each TN domain and then take the average of all domains. Results are depicted in Fig. 6. Here, horizontal and vertical coordinates represent the amplitudes of light in $x$ and $y$ directions. Black dotted lines show the incident light, linearly polarized in $x$ direction. The outgoing light (the solid blue line) is elliptically polarized, but the eccentricity of this ellipse is close to unity, so it looks like a linear polarization with polarization direction rotated by $4.5^{\circ}$. So the ORP in the thin TN model is defined as the rotation angle of the ellipse's long axis divided by the cell gap. Based on simulation result, this ORP is described by

$$
\varphi=\varphi_{o} \frac{(\Delta n)^{2}}{\lambda^{2} / \lambda_{B}^{2}},
$$

where $\varphi_{0}$ is a constant, $\Delta n$ is the LC birefringence, and $\lambda_{B}$ is the Bragg reflection wavelength of this cell, determined by the refractive index and the thickness of each TN layer. Note that Eq. (4) is quite similar to Eq. (3) except for the denominator. Actually, Eq. (3) is reduced to Eq. (4) when $\lambda_{B} \ll \lambda$. The physical meaning of this approximation is when the Bragg reflection wavelength is much shorter than the incident wavelength, Bragg reflection is negligible. Under this approximation, the optical activity of BPLC could be explained by this thin TN model.

In conclusion, we have investigated the polarization rotation of polymer-stabilized BPLCs. The effects of birefringence, Bragg reflection wavelength, and incident light wavelength on the ORP of BPLC are evaluated. It is shown that by correcting the ORP, the device contrast ratio can be improved by more than $3 \times$. A thin TN model is proposed to explain the polarization rotation phenomenon. Simulation

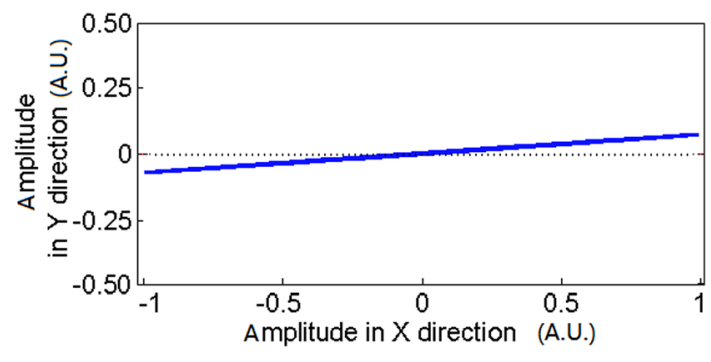

FIG. 6. Polarization state change of the light passing through a multidomain multi-layer TN cell: black dotted lines show the incident polarization state. Blue solid line shows the simulated outgoing polarization state.

result based on this model is found to be an approximation of experimental results when $\lambda_{\mathrm{B}} \ll \lambda$.

The authors are indebted to Jie Sun, Jin Yan, and Yuan Chen for useful discussion and AU Optronics (Taiwan) for financial support.

${ }^{1}$ H. Kikuchi, M. Yokota, Y. Hisakado, H. Yang, and T. Kajiyama, Nature Mater. 1, 64 (2002).

${ }^{2}$ S. W. Choi, S. I. Yamamoto, Y. Haseba, H. Higuchi, and H. Kikuchi, Appl. Phys. Lett. 92, 043119 (2008).

${ }^{3}$ Z. Ge, S. Gauza, M. Jiao, H. Xianyu, and S. T. Wu, Appl. Phys. Lett. 94, 101104 (2009).

${ }^{4}$ J. Yan, L. Rao, M. Jiao, Y. Li, H. C. Cheng, and S. T. Wu, J. Mater. Chem. 21, 7870 (2011).

${ }^{5}$ H. Lee, H. J. Park, O. J. Kwon, S. J. Yun, J. H. Park, S. Hong, and S. T. Shin, SID Int. Symp. Digest Tech. Papers 42, 121 (2011).

${ }^{6}$ K. M. Chen, S. Gauza, H. Xianyu, and S. T. Wu, J. Display Technol. 6, 49 (2010).

${ }^{7}$ Y. Chen, J. Yan, J. Sun, S. T. Wu, X. Liang, S. H. Liu, P. J. Hsieh, K. L. Cheng, and J. W. Shiu, Appl. Phys. Lett. 99, 201105 (2011).

${ }^{8}$ L. Rao, Z. Ge, S. T. Wu, and S. H. Lee, Appl. Phys. Lett. 95, 231101 (2009).

${ }^{9}$ L. Rao, J. Yan, S. T. Wu, S. Yamamoto, and Y. Haseba, Appl. Phys. Lett. 98, 081109 (2011).

${ }^{10}$ M. Wittek, N. Tanaka, D. Wilkes, M. Bremer, D. Pauluth, J. Canisius, A. Yeh, R. Yan, K. Skjonnemand, and M. Klasen-Memmer, SID Int. Symp. Digest Tech. Papers 43, 25 (2012).

${ }^{11}$ K. M. Chen, S. Gauza, H. Xianyu, and S. T. Wu, J. Display Technol. 6, 318 (2010).

${ }^{12}$ L. Rao, J. Yan, S. T. Wu, Y. H. Chiu, H. Y. Chen, C. C. Liang, C. M. Wu, P. J. Hsieh, S. H. Liu, and K. L. Cheng, J. Display Technol. 7, 627 (2011).

${ }^{13}$ Y. Hirakata, D. Kubota, A. Yamashita, T. Ishitani, T. Nishi, H. Miyake, H. Miyairi, J. Koyama, S. Yamazaki, T. Cho, and M. Sakakura, J. Soc. Info. Display 20, 38 (2012).

${ }^{14}$ S. Yoon, G.-H. Yang, P. Nayek, H. Jeong, S.-H. Lee, S.-H. Hong, H.-J. Lee, and S.-T. Shin, J. Phys. D: Appl. Phys. 45, 105304 (2012).

${ }^{15}$ D. Bensimon, E. Domany, and S. Shtrikman, Phys. Rev. A 28, 427 (1983).

${ }^{16}$ V. A. Belyakov and V. E. Dmitrienko, Sov. Phys. Usp. 28, 535 (1985).

${ }^{17}$ Z. Kutnjak, C. W. Garland, C. G. Schatz, P. J. Collings, C. J. Booth, and J. W. Goodby, Phys. Rev. E 53, 4955 (1996).

${ }^{18}$ B.-Y. Zhang, F.-B. Meng, and Y.-H. Cong, Opt. Express 15, 10175 (2007).

${ }^{19}$ S. Meiboom, J. P. Sethna, W. P. Anderson, and W. F. Brinkman, Phys. Rev. Lett. 46, 1216 (1981).

${ }^{20}$ M. Schadt and W. Helfrich, Appl. Phys. Lett. 18, 127 (1971).

${ }^{21}$ J. Yan and S. T. Wu, Opt. Mater. Express 1, 1527 (2011).

${ }^{22}$ H. C. Cheng, J. Yan, T. Ishinabe, and S. T. Wu, Appl. Phys. Lett. 98, 261102 (2011).

${ }^{23}$ J. Yan, Y. Chen, S. T. Wu, and X. Song, J. Display Technol. 9, 24 (2013).

${ }^{24}$ S. T. Wu and C. S. Wu, Phys. Rev. A 42, 2219 (1990).

${ }^{25}$ J. Yan, H. C. Cheng, S. Gauza, Y. Li, M. Jiao, L. Rao, and S. T. Wu, Appl. Phys. Lett. 96, 071105 (2010).

${ }^{26}$ H. Baessler, T. M. Laronge, and M. M. Labes, J. Chem. Phys. 51, 3213 (1969). 